\title{
1-absorbing primary submodules
}

\author{
Ece Yetkin Celikel
}

\begin{abstract}
Let $R$ be a commutative ring with non-zero identity and $M$ be a unitary $R$-module. The goal of this paper is to extend the concept of 1 absorbing primary ideals to 1-absorbing primary submodules. A proper submodule $N$ of $M$ is said to be a 1-absorbing primary submodule if whenever non-unit elements $a, b \in R$ and $m \in M$ with $a b m \in N$, then either $a b \in\left(N:_{R} M\right)$ or $m \in M-\operatorname{rad}(N)$. Various properties and chacterizations of this class of submodules are considered. Moreover, 1-absorbing primary avoidance theorem is proved.
\end{abstract}

\section{Introduction}

Throughout this paper, we shall assume unless otherwise stated, that all rings are commutative with non-zero identity and all modules are considered to be unitary. A prime (resp. primary) submodule is a proper submodule $N$ of $M$ with the property that for $a \in R$ and $m \in M$, am $\in N$ implies that $m \in N$ or $a \in\left(N:_{R} M\right)\left(\right.$ resp. $\left.a \in \sqrt{\left(N:_{R} M\right)}\right)$. Since prime and primary ideals (submodules) have an important role in the theory of modules over commutative rings, generalizations of these concepts have been studied by several authors [1]-[8], [14], [15]. For a survey article consisting some of generalizations see [5]. In 2007, Badawi [4] called a non-zero proper ideal $I$ of $R$ a 2-absorbing ideal of $R$ if whenever $a, b, c \in R$ and $a b c \in I$, then $a b \in I$ or $a c \in I$ or $b c \in I$. As an extension of 2-absorbing primary ideals, the concept of 2-absorbing submodules are introduced by Darani and Soheilnia [8] and studied by Payrovi,

Key Words: 1-absorbing primary submodule, 1-absorbing primary ideal, 2-absorbing primary submodule

2010 Mathematics Subject Classification: Primary 13C05, Secondary 13C99.

Received: 03.01.2021

Accepted: 28.04.2021 
Babaei [15]. We recall from [8] that a proper submodule $N$ of $M$ is said to be a 2-absorbing submodule if whenever $a, b \in R$ and $m \in M$ with $a b m \in N$, then $a b \in\left(N:_{R} M\right)$ or $a m \in N$ or $b m \in N$. In 2014, Badawi, Tekir and Yetkin [6] introduced the concept of 2-absorbing primary ideals. A proper ideal $I$ of $R$ is called 2-absorbing primary if whenever $a, b, c \in R$ with $a b c \in I$, then $a b \in I$ or $a c \in \sqrt{I}$ or $b c \in \sqrt{I}$. After that, the notion of 2-absorbing pimary submodules is introduced and studied in [14]. According to [14], a proper submodule $N$ of $M$ is said to be 2-absorbing primary provided that $a, b \in R, m \in M$ and $a b m \in N$ imply either $a b \in\left(N:_{R} M\right)$ or $a m \in M-\operatorname{rad}(N)$ or $b m \in M-\operatorname{rad}(N)$. As a recent study, the class of 1-absorbing primary ideals was defined in [7]. According to [7], a proper ideal $I$ of $R$ is said to be a 1-absorbing primary ideal if whenever non-unit elements $a, b, c$ of $R$ and $a b c \in I$, then $a b \in I$ or $c \in \sqrt{I}$. Our aim is to extend the notion of 1-absorbing primary ideals to 1-absorbing primary submodules.

For the sake of thoroughness, we give some definitions which we will need throughout this study. Let $I$ be an ideal of a ring $R$. By $\sqrt{I}$, we mean the radical of $I$ which is the intersection of all prime ideals containing $I$, that is $\left\{r \in R: r^{n} \in I\right.$ for some $\left.n\right\}$. Let $M$ be an $R$-module and $N$ be a submodule of $M$. We will denote by $\left(N:_{R} M\right)$ the residual of $N$ by $M$, that is, the set of all $r \in R$ such that $r M \subseteq N$. The annihilator of $M$ denoted by $A n n_{R}(M)$ is $\left(0:_{R} M\right)$. The $M$-radical of $N$, denoted by $M-\operatorname{rad}(N)$, is defined to be the intersection of all prime submodules of $M$ containing $N$. If $M$ is a multiplication $R$-module, then $M-\operatorname{rad}(N)=\left\{m \in M: m^{k} \subseteq N\right.$ for some $k \geq 0\}$ [1, Theorem 3.13]. If there is no such a prime submodule, then $M-\operatorname{rad}(N)=M$. For the other notations and terminologies that are used in this article, the reader is referred to [10].

We summarize the content of this article as follows. We call a proper submodule $N$ of $M$ a 1-absorbing primary submodule if whenever non-unit elements $a, b \in R$ and $m \in M$ with $a b m \in N$, then $a b \in\left(N:_{R} M\right)$ or $m \in M-\operatorname{rad}(N)$. It is clear that a prime submodule is a 1-absorbing primary submodule, and a 1-absorbing primary submodule is a 2-absorbing primary submodule. In Section 2, we start with examples (Example 1 and Example 2) showing that the inverses of these implications are not true in general. Various characterizations for 1-absorbing primary submodules are given (Theorem 1, Theorem 2 and Theorem 3). Moreover, the behavior of 1-absorbing primary submodules in modules under homomorphism, module localizations and direct product of modules are investigated (Proposition 3, Proposition 4 and Proposition 5). Finally, in Section 3, the 1-absorbing primary avoidance theorem is proved. 


\section{Properties of 1 -absorbing primary submodules}

Definition 1. Let $M$ be a module over a commutative ring $R$ and $N$ be a proper submodule of $M$. We call $N$ a 1-absorbing primary submodule if whenever non-unit elements $a, b \in R$ and $m \in M$ with abm $\in N$, then $a b \in$ $\left(N:_{R} M\right)$ or $m \in M-\operatorname{rad}(N)$.

It is clear that the following implication hold: prime submodule $\Rightarrow 1$ absorbing primary submodule $\Rightarrow 2$-absorbing primary submodule. The following example shows that a 1-absorbing primary submodule of $M$ needs not to be a primary (prime) submodule; and also there are 2-absorbing primary submodules which are not 1-absorbing primary.

Example 1. 1. Let $A=K[x, y]$, where $K$ is a field, $Q=(x, y) A$. Consider $R=A_{Q}$ and $M=R$ as an $R$-module. Then $N=\left(x^{2}, x y\right) M$ is a 1-absorbing primary submodule of $M$ [7, Example 1]. Observe that $\sqrt{\left(N:_{R} M\right)}=x R$. Since $x \cdot y \in N$, but $x \notin N$ and $y \notin \sqrt{\left(N:_{R} M\right)}, N$ is not a primary submodule (so, it is not a prime submodule) of $M$.

2. Consider the submodule $N=p^{2} q \mathbb{Z}$ of $\mathbb{Z}$-module $\mathbb{Z}$ where $p$ and $q$ are distinct prime integers. Then $N$ is a 2-absorbing primary submodule of $\mathbb{Z}$ by [14, Corollary 2.21]. However it is not 1-absorbing primary as $p \cdot p \cdot q \in N$ but neither $p \cdot p \in(N: \mathbb{Z} \mathbb{Z})=N$ nor $q \in M-\operatorname{rad}(N)=p q \mathbb{Z}$.

The following example shows that there are some modules of which every proper submodule is 2-absorbing primary but which has no 1-absorbing primary submodule.

Example 2. Let $p$ be a fixed prime integer. Then $E(p)=\{\alpha \in \mathbb{Q} / \mathbb{Z}: \alpha=$ $r / p^{n}+\mathbb{Z}$ for some $r \in \mathbb{Z}$ and $\left.n \in \mathbb{N} \cup(0)\right\}$ is a non-zero submodule of $\mathbb{Z}$-module $\mathbb{Q} / \mathbb{Z}$. For each $t \in \mathbb{N}$, set $G_{t}=\left\{\alpha \in \mathbb{Q} / \mathbb{Z}: \alpha=r / p^{t}+Z\right.$ for some $\left.r \in \mathbb{Z}\right\}$. Observe that each proper submodule of $E(p)$ is equal to $G_{i}$ for some $i \in \mathbb{N}$ and $\left(G_{t}:_{\mathbb{Z}} E(p)\right)=0$ for every $t \in \mathbb{N}$. It is shown in [3, Example 1] that every submodule $G_{t}$ is not a primary submodule of $E(p)$. Thus there is no prime submodule in $E(p)$. Thus $E(p)-\operatorname{rad}\left(G_{t}\right)=E(p)$. Therefore, each $G_{t}$ is a 1-absorbing primary submodule of $R$.

We next give several characterizations of 1-absorbing primary submodules of an $R$-module.

Theorem 1. Let $N$ be a proper submodule of an $R$-module $M$. Then the following statements are equivalent:

1. $N$ is a 1-absorbing primary submodule of $M$. 
2. If $a, b$ are non-unit elements of $R$ such that $a b \notin\left(N:_{R} M\right)$, then $\left(N:_{M}\right.$ $a b) \subseteq M-\operatorname{rad}(N)$.

3. If $a, b$ are non-unit elements of $R$, and $K$ is a submodule of $M$ with $a b K \subseteq N$, then $a b \in\left(N:_{R} M\right)$ or $K \subseteq M-\operatorname{rad}(N)$.

4. If $I_{1} I_{2} K \subseteq N$ for some proper ideals $I_{1}, I_{2}$ of $R$ and some submodule $K$ of $M$, then either $I_{1} I_{2} \subseteq\left(N:_{R} M\right)$ or $K \subseteq M-\operatorname{rad}(N)$.

Proof. $(1) \Rightarrow(2)$ Suppose that $a, b$ are non-unit elements of $R$ such that $a b \notin$ $\left(N:_{R} M\right)$. Let $m \in\left(N:_{M} a b\right)$. Hence $a b m \in N$. Since $N$ is 1-absorbing primary submodule and $a b \notin\left(N:_{R} M\right)$, we have $m \in M-\operatorname{rad}(N)$, and so $(N: M a b) \subseteq M-\operatorname{rad}(N)$.

$(2) \Rightarrow(3)$ Suppose that $a b \notin\left(N:_{R} M\right)$. Since $a b K \subseteq N$, we have $K \subseteq$ $\left(N:_{M} a b\right) \subseteq M-\operatorname{rad}(N)$ by $(2)$.

$(3) \Rightarrow(4)$ Assume on the contrary that neither $I_{1} I_{2} \subseteq\left(N:_{R} M\right)$ nor $K \subseteq$ $M-\operatorname{rad}(N)$. Then there exist non-unit elements $a \in I_{1}, b \in I_{2}$ with $a b \notin$ $\left(N:_{R} M\right)$. Thus $a b K \subseteq N$, it contradicts with (3).

$(4) \Rightarrow(1)$ Let $a, b \in R$ be non-unit elements, $m \in M$ and $a b m \in N$. Put $I_{1}=a R, I_{2}=b R, K=R m$. Thus the result is clear.

An $R$-module $M$ is called a multiplication module if every submodule $N$ of $M$ has the form $I M$ for some ideal $I$ of $R$. Equivalently, $N=\left(N:_{R} M\right) M$ [9]. Let $M$ be a multiplication $R$-module and let $N=I M$ and $K=J M$ for some ideals $I$ and $J$ of $R$. The product of $N$ and $K$ is denoted by $N K$ is defined by $I J M$. Clearly, $N K$ is a submodule of $M$ and contained in $N \cap K$. It is shown in [1, Theorem 3.4] that the product of $N$ and $K$ is independent of presentations of $N$ and $K$. It is shown in [9, Theorem 2.12] that if $N$ is a proper submodule of a multiplication $R$-module $M$, then $M-\operatorname{rad}(N)=\sqrt{\left(N:_{R} M\right)} M$. If $M$ is a finitely generated multiplication $R$-module, then $(M-\operatorname{rad}(N): M)=$ $\sqrt{\left(N:_{R} M\right)}$ by $[14$, Lemma 2.4]. Now, we are ready for characterizing 1 absorbing primary submodules of finitely generated multiplication module $M$ in terms of submodules of $M$.

Theorem 2. Let $M$ be a finitely generated multiplication $R$-module and $N$ be a proper submodule of $M$. Then the following statements are equivalent:

1. $N$ is a 1-absorbing primary submodule of $M$.

2. If $N_{1} N_{2} N_{3} \subseteq N$ for some submodules $N_{1}, N_{2}, N_{3}$ of $M$, then either $N_{1} N_{2} \subseteq N$ or $N_{3} \subseteq M-\operatorname{rad}(N)$.

Proof. $(1) \Rightarrow(2)$ Suppose that $N$ is a 1-absorbing primary submodule of $M$, $N_{1} N_{2} N_{3} \subseteq N$ and $N_{3} \nsubseteq M-\operatorname{rad}(N)$. Since $M$ is a finitely generated multiplication module, $N_{1}=I_{1} M$ and $N_{2}=I_{2} M$ for some ideals $I_{1}, I_{2}$ of $R$. Hence 
$I_{1} I_{2} N_{3} \subseteq N$. Since $N_{3} \nsubseteq \subseteq M-\operatorname{rad}(N)$, we have $I_{1} I_{2} \subseteq\left(N:_{R} M\right)$ by Theorem 1. Thus we conclude $N_{1} N_{2} \subseteq(N: M) M=N$.

$(2) \Rightarrow(1)$ Let $I_{1} I_{2} K \subseteq N$. Then there exists an ideal $I_{3}$ of $R$ such that $I_{1} I_{2} I_{3} M \subseteq N$ which gives $I_{1} I_{2} M \subseteq N$ or $I_{3} M \subseteq M-\operatorname{rad}(N)$. By [16, p.231 Corollary], we have $I_{1} I_{2} \subseteq\left(N:_{R} M\right)+A n n_{R}(M)=\left(N:_{R} M\right)$ or $K \subseteq M-\operatorname{rad}(N)$. Hence, we are done from Theorem 1 .

Lemma 1. [16, Theorem 10]Let $M$ be a finitely generated faithful multiplication $R$-module, then $(I M: M)=I$.for all ideals $I$ of $R$.

In [3, Corollary 2], for a proper submodule $N$ of a multiplication $R$-module $M$, it is shown that $N$ is primary submodule of $M$ if and only if $\left(N:_{R} M\right)$ is primary ideal of $R$. Analogous with this result, we have the following.

Theorem 3. Let I be an ideal of a ring $R$ and $N$ be a submodule of a finitely generated faithful multiplication $R$-module $M$. Then

1. $I$ is a 1-absorbing primary ideal of $R$ if and only if $I M$ is a 1-absorbing primary submodule of $M$.

2. $N$ is a 1-absorbing primary submodule of $M$ if and only if $(N: M)$ is a 1-absorbing primary ideal of $R$.

3. $N$ is a 1-absorbing primary submodule of $M$ if and only if $N=I M$ for some 1-absorbing primary ideal of $R$.

Proof. (1) Suppose $I$ is a 1-absorbing primary ideal of $R$. If $I M=M$, then $I=(I M: M)=R$ by Lemma 1, a contradiction. Thus, $I M$ is proper in $M$. Now, let $a, b \in R$ be non-unit elements and $m \in M$ such that abm $\in I M$ and $a b \notin(I M: M)=I$. Then $a b((m): M)=((a b m): M) \subseteq(I M:$ $M) \subseteq(\sqrt{I} M: M)=\sqrt{I}$. Since $I$ is a 1 -absorbing primary ideal, we conclude that $((m): M) \subseteq \sqrt{I}$. Thus, $m \in((m): M) M \subseteq \sqrt{I} M=M-\operatorname{rad}(I M)$. Conversely, suppose $I M$ is 1-absorbing primary submodule of $M$. Then clearly $I$ is proper in $R$. Let $a, b, c \in R$ be non-unit elements with $a b c \in I$ and $a b \notin$ $(I M: M)=I$. Since $a b M \in I M$ and $I M$ is a 1-absorbing primary submodule, then $c M \subseteq M-\operatorname{rad}(I M)=\sqrt{I} M$. Therefore, $c \in(\sqrt{I} M: M)=\sqrt{I}$ and $I$ is a 1-absorbing primary ideal of $R$.

(2) Since $N=(N: M) M$, it follows by (1).

(3) Putting $I=(N: M)$ in (2), the claim is clear.

The following example shows that if $\left(N:_{R} M\right)$ is a 1-absorbing primary ideal of $R$, then $N$ is not needed to be a 1-absorbing primary submodule in general. 
Example 3. Let $R=\mathbb{Z}$ and $M=\mathbb{Z} \times \mathbb{Z}$ be an $R$-module and $p$ a prime integer. Consider the submodule $N=p^{n} \mathbb{Z} \times\{0\}$ of $M$ for $n \geq 2$. Then $\left(N:_{R} M\right)=\{0\}$ is a 1-absorbing primary ideal of $R$. However, $N$ is not a 1-absorbing primary submodule of $M$ since $p \cdot p^{n-1} \cdot(1,0) \in N$ but neither $p \cdot p^{n-1}=p^{n} \in\left(N:_{R} M\right)=\{0\} \operatorname{nor}(1,0) \in M-\operatorname{rad}(N)=p \mathbb{Z} \times\{0\}$.

In view of Theorem 3 , we conclude the following result.

Proposition 1. Let $M$ be a finitely generated multiplication $R$-module and $N$ be a 1-absorbing primary submodule of $M$. Then the following are satisfied:

1. $\sqrt{\left(N:_{R} M\right)}$ is a prime ideal of $R$.

2. $\sqrt{\left(N:_{R} m\right)}$ is a prime ideal of $R$ containing $\sqrt{\left(N:_{R} M\right)}=P$ for every $m \notin M-\operatorname{rad}(N)$.

3. $M-\operatorname{rad}(N)$ is a prime submodule of $M$.

Proof. (1) Let $N$ be a 1-absorbing primary submodule of $M$. Then $\left(N:_{R} M\right)$ is 1-absorbing primary ideal of $R$ by Theorem 3. From [7, Theorem 2], we conclude that $\sqrt{\left(N:_{R} M\right)}$ is a prime ideal of $R$.

(2) Since $N$ is a 1-absorbing primary ideal, $\sqrt{\left(N:_{R} M\right)}=P$ is a prime ideal of $R$ by (1). Suppose that $a, b \in R$ such that $a b \in \sqrt{\left(N:_{R} m\right)}$. Without loss of generality we may assume that $a$ and $b$ are non-unit elements of $R$. Then there exists a positive integer $n$ such that $a^{n} b^{n} m \in N$. Since $N$ is 1absorbing primary submodule, and $m \notin M-\operatorname{rad}(N)$, it implies that either $(a b)^{n} \in\left(N:_{R} M\right)$. Since $P$ is prime and $a b \in P$, we conclude either $a \in P=$ $\sqrt{\left(N:_{R} M\right)} \subseteq \sqrt{\left(N:_{R} m\right)}$ or $b \in P=\sqrt{\left(N:_{R} M\right)} \subseteq \sqrt{\left(N:_{R} m\right)}$.

(3) Suppose thet $N$ is a 1-absorbing primary submodule. Since $\sqrt{\left(N:_{R} M\right)}$ is a prime ideal of $R$ by (1), we conclude that $M-\operatorname{rad}(N)=\sqrt{\left(N:_{R} M\right)} M$ is a prime submodule of $M$ by [9, Corollary 2.11].

Note that the intersection of two distinct non-zero 1-absorbing primary submodules need not be a 1-absorbing primary submodule. Consider $\mathbb{Z}$ module $\mathbb{Z}$. Then $2 \mathbb{Z}$ and $3 \mathbb{Z}$ are clearly 1 -absorbing primary submodules but $2 \mathbb{Z} \cap 3 \mathbb{Z}=6 \mathbb{Z}$ is not. Indeed, $2 \cdot 2 \cdot 3 \in 6 \mathbb{Z}$ but neither $2 \cdot 2 \in(\mathbb{Z}: 6 \mathbb{Z})=6 \mathbb{Z}$ nor $3 \in \mathbb{Z}-\operatorname{rad}(6 \mathbb{Z})=6 \mathbb{Z}$. We call a proper submodule $N$ of $M$ a $P-1$ absorbing submodule of $M$ if $\sqrt{\left(N:_{R} M\right)}=P$ is a prime submodule of $R$. In the next theorem, we show that if $N_{i}$ 's are $P$-1-absorbing primary submodules of a multiplication module $M$, then the intersection of these submodules is a $P$-1-absorbing primary submodule of $M$. 
Proposition 2. Let $M$ be a multiplication R-module. If $\left\{N_{i}\right\}_{i=1}^{k}$ is a family of P-1-absorbing primary submodules of $M$, then so is $\bigcap_{i=1}^{k} N_{i}$.

Proof. Suppose that $a b m \in \bigcap_{i=1}^{k} N_{i}$ but $a b \notin\left(\bigcap_{i=1}^{k} N_{i}:_{R} M\right)$ for non-unit elements $a, b \in R$ and $m \in M$. Then $a b \notin\left(N_{j}:_{R} M\right)$ for some $j \in\{1, \ldots, k\}$. Since $N_{j}$ is 1-absorbing primary and $a b m \in N_{j}$, we have $m \in M-\operatorname{rad}\left(N_{j}\right)$. Now, since $M-\operatorname{rad}\left(\bigcap_{i=1}^{k} N_{i}\right)=\bigcap_{i=1}^{k} M-\operatorname{rad}\left(N_{i}\right)=P M$ by $[14$, Proposition $2.14(3)]$, we are done.

Lemma 2. [12]Let $\varphi: M_{1} \longrightarrow M_{2}$ be an R-module epimorphism. Then

1. If $N$ is a submodule of $M_{1}$ and $\operatorname{ker}(\varphi) \subseteq N$, then $\varphi\left(M_{1}-\operatorname{rad}(N)\right)=M_{2}$ $\operatorname{rad}(\varphi(N))$.

2. If $K$ is a submodule of $M_{2}$, then $\varphi^{-1}\left(M_{2}-\operatorname{rad}(K)\right)=M_{1}-\operatorname{rad}\left(\varphi^{-1}(K)\right)$.

Proposition 3. Let $M_{1}$ and $M_{2}$ be $R$-modules and $f: M_{1} \rightarrow M_{2}$ be a module homomorphism. Then the following statements hold:

1. If $N_{2}$ is a 1-absorbing primary submodule of $M_{2}$, then $f^{-1}\left(N_{2}\right)$ is a 1-absorbing primary submodule of $M_{1}$.

2. Let $f$ be an epimorphism. If $N_{1}$ is a 1-absorbing primary submodule of $M_{1}$ containing $\operatorname{Ker}(f)$, then $f\left(N_{1}\right)$ is a 1-absorbing primary submodule of $M_{2}$.

Proof. (1) Suppose that $a, b$ are non-unit elements of $R, m_{1} \in M_{1}$ and $a b m_{1} \in$ $f^{-1}\left(N_{2}\right)$. Then $\operatorname{abf}\left(m_{1}\right) \in N_{2}$. Since $N_{2}$ is 1-absorbing primary, we have either $a b \in\left(N_{2}:_{R} M_{2}\right)$ or $f\left(m_{1}\right) \in M_{2}-\operatorname{rad}\left(N_{2}\right)$. Here, we show that $\left(N_{2}:_{R}\right.$ $\left.M_{2}\right) \subseteq\left(f^{-1}\left(N_{2}\right):_{R} M_{1}\right)$. Let $r \in\left(N_{2}:_{R} M_{2}\right)$. Then $r M_{2} \subseteq N_{2}$ which implies that $r f^{-1}\left(M_{2}\right) \subseteq f^{-1}\left(N_{2}\right)$; i.e. $r M_{1} \subseteq f^{-1}\left(N_{2}\right)$. Thus $r \in\left(f^{-1}\left(N_{2}\right):_{R} M_{1}\right)$. Hence $a b \in\left(f^{-1}\left(N_{2}\right):_{R} M_{1}\right)$ or $m_{1} \in f^{-1}\left(M_{2}-\operatorname{rad}\left(N_{2}\right)\right)$. Since $f^{-1}\left(M_{2}-\right.$ $\left.\operatorname{rad}\left(N_{2}\right)\right)=M_{1}-\operatorname{rad}\left(f^{-1}\left(N_{2}\right)\right)$ by Lemma $2(2)$ and $f^{-1}\left(N_{2}\right)$ is a 1-absorbing primary submodule of $M_{1}$.

(2) Suppose that $a, b$ are non-unit elements of $R, m_{2} \in M_{2}$ and $a b m_{2} \in$ $f\left(N_{1}\right)$. Since $f$ is an epimorphism, there exists $m_{1} \in M_{1}$ such that $f\left(m_{1}\right)=$ $m_{2}$. Since $\operatorname{Ker} f \subseteq N_{1}, a b m_{1} \in N_{1}$. Hence $a b \in\left(N_{1}:_{R} M_{1}\right)$ or $m_{1} \in M_{1}-$ $\operatorname{rad}\left(N_{1}\right)$. Here, we show that $\left(N_{1}:_{R} M_{1}\right) \subseteq\left(f\left(N_{1}\right):_{R} M_{2}\right)$. Let $r \in\left(N_{1}:_{R}\right.$ $\left.M_{1}\right)$. Then $r M_{1} \subseteq N_{1}$ which implies that $r f\left(M_{1}\right) \subseteq f\left(N_{1}\right)$. Since $f$ is 
onto, we conclude that $r M_{2} \subseteq f\left(N_{1}\right)$, that is, $r \in\left(f\left(N_{1}\right):_{R} M_{2}\right)$. Thus $a b \in\left(f\left(N_{1}\right):_{R} M_{2}\right)$ or $m_{2}=f\left(m_{1}\right) \in f\left(M_{1}-\operatorname{rad}\left(N_{1}\right)\right)=M_{2}-\operatorname{rad}\left(f\left(N_{1}\right)\right)$ by Lemma 2 (1), as desired.

As a consequence of Theorem 3, we have the following result.

Corollary 1. Let $M$ be an $R$-module and $N_{1}, N_{2}$ be submodules of $M$ with $N_{2} \subseteq N_{1}$. Then $N_{1}$ is a 1-absorbing primary submodule of $M$ if and only if $N_{1} / N_{2}$ is a 1-absorbing primary submodule of $M / N_{2}$.

Proof. Suppose that $N_{1}$ is a 1-absorbing primary submodule of $M$. Consider the canonical epimorphism $f: M \rightarrow M / N_{2}$ in Proposition 3. Then $N_{1} / N_{2}$ is a 1-absorbing primary submodule of $M / N_{2}$. Conversely, let $a$ and $b$ are nonunit elements of $R, m \in M$ such that $a b m \in N_{1}$. Hence $a b\left(m+N_{2}\right) \in N_{1} / N_{2}$. Since $N_{1} / N_{2}$ is a 1-absorbing primary submodule of $M / N_{2}$, it implies either $a b \in\left(N_{1} / N_{2}:_{R} M / N_{2}\right)$ or $m+N_{2} \in M / N_{2}-\operatorname{rad}\left(N_{1} / N_{2}\right)=M-\operatorname{rad}\left(N_{1}\right) / N_{2}$. Therefore $a b \in\left(N_{1}:_{R} M\right)$ or $m \in M-\operatorname{rad}\left(N_{1}\right)$. Thus $N_{1}$ is a 1-absorbing primary submodule of $M$.

Let $M_{1}$ be $R_{1}$-module and $M_{2}$ be $R_{2}$-module where $R_{1}$ and $R_{2}$ are commutative rings with identity. Let $R=R_{1} \times R_{2}$ and $M=M_{1} \times M_{2}$. Then $M$ is an $R$-module and every submodule of $M$ is of the form $N=N_{1} \times N_{2}$ for some submodules $N_{1}, N_{2}$ of $M_{1}, M_{2}$, respectively. Also, $M-\operatorname{rad}\left(N_{1} \times N_{2}\right)=$ $M_{1}-\operatorname{rad}\left(N_{1}\right) \times M_{2}-\operatorname{rad}\left(N_{2}\right)$ by $[2, \operatorname{Lemma} 2.3$ (ii)].

Proposition 4. Let $M_{1}$ be an $R_{1}$-module and $M_{2}$ be an $R_{2}$-module, where $R_{1}, R_{2}$ are commutative rings with identity, $R=R_{1} \times R_{2}$ and $M=M_{1} \times$ $M_{2}$. Suppose that $N_{1}$ is a proper submodule of $M_{1}$. If $N=N_{1} \times M_{2}$ is a 1-absorbing primary submodule of $R$-module $M$, then $N_{1}$ is a 1-absorbing primary submodule of $R_{1}$-module $M_{1}$.

Proof. Suppose that $N=N_{1} \times M_{2}$ is a 1-absorbing primary submodule of $M$. Put $M^{\prime}=M /\{0\} \times M_{2}$ and $N^{\prime}=N /\{0\} \times N_{2}$. From Corollary $1, N^{\prime}$ is a 1-absorbing primary submodule of $M^{\prime}$. Since $M^{\prime} \cong M_{1}$ and $N^{\prime} \cong N_{1}$, we conclude the result.

Proposition 5. Let $S$ be a multiplicatively closed subset of a commutative ring $R$ and $M$ be an $R$-module. If $N$ is a 1-absorbing primary submodule of $M$ and $S^{-1} N \neq S^{-1} M$, then $S^{-1} N$ is a 1-absorbing primary submodule of $S^{-1} R$-module $S^{-1} M$.

Proof. Let $\frac{a}{s_{1}}$ and $\frac{b}{s_{2}}$ be non-unit elements of $S^{-1} R, \frac{m}{s_{3}} \in S^{-1} M$ with $\frac{a}{s_{1}} \frac{b}{s_{2}} \frac{m}{s_{3}} \in$ $S^{-1} N$. Hence tabm $\in N$ for some $t \in S$. Since $N$ is 1-absorbing primary, we have either $t m \in M-\operatorname{rad}(N)$ or $a b \in\left(N:_{R} M\right)$. Thus we conclude either 
$\frac{m}{s_{3}}=\frac{t m}{t s_{3}} \in S^{-1}(M-\operatorname{rad}(N)) \subseteq S^{-1} M-\operatorname{rad}\left(S^{-1} N\right)$ or $\frac{a b}{s_{1} s_{2}} \in S^{-1}\left(N:_{R}\right.$ $M) \subseteq\left(S^{-1} N: S_{S^{-1} R} S^{-1} M\right)$.

Let $R$ be a ring and $M$ be an $R$-module. The idealization of $M$ is denoted by $R(M)=R(+) M$ is a commutative ring with identity with coordinate-wise addition and multiplication defined by $\left(a, m_{1}\right)\left(b, m_{2}\right)=\left(a b, a m_{2}+b m_{1}\right)$. An ideal $H$ is called homogeneous if $H=I(+) N$ for some ideal $I$ of $R$ and some submodule $N$ of $M$ such that $I M \subseteq N$.

Proposition 6. Let $M$ be an R-module and $I(+) N$ be a homogeneous ideal of $R(M)$. If $I(+) N$ is a 1-absorbing primary ideal of $R(M)$, then $I$ is a 1 absorbing primary ideal of $R$.

Proof. Suppose that $a, b, c$ are non-unit elements of $R$ such that $a b c \in I$ and $c \notin \sqrt{I}$. Then $\left(a, 0_{M}\right) \cdot\left(b, 0_{M}\right) \cdot\left(c, 0_{M}\right) \in I(+) N$. Note that $\sqrt{I(+) N}=$ $\sqrt{I}(+) M$ by $[10$, Theorem $25.1(5)]$. Then $\left(c, 0_{M}\right) \notin \sqrt{I(+) N}$. Since $I(+) N$ is 1-absorbing primary, we conclude that $\left(a, 0_{M}\right) \cdot\left(b, 0_{M}\right) \in I(+) N$. Thus $a b \in I$, we are done.

\section{The 1-absorbing primary avoidance theorem}

In this section, we prove the 1-absorbing primary avoidance theorem. Throughout this section, let $M$ be a finitely generated multiplication $R$-module and $N, N_{1}, \ldots, N_{n}$ be submodules of $M$. Recall from [11] that a covering $N \subseteq$ $N_{1} \cup N_{2} \cup \cdots \cup N_{n}$ is said to be efficient if no $N_{k}$ is superfluous. Also, $N=N_{1} \cup N_{2} \cup \cdots \cup N_{n}$ is an efficient union if none of the $N_{k}$ may be excluded. A covering of a submodule by two submodules is never efficient.

Theorem 4. Let $N \subseteq N_{1} \cup N_{2} \cup \cdots \cup N_{n}$ be an efficient covering of submodules $N_{1}, N_{2}, \ldots, N_{n}$ of $M$ where $n>2$. If $\sqrt{\left(N_{i}:_{R} M\right)} \nsubseteq \sqrt{\left(N_{j}:_{R} m\right)}$ for all $m \in$ $M \backslash M-\operatorname{rad}\left(N_{j}\right)$ whenever $i \neq j$, then no $N_{i}(1 \leq i \leq n)$ is a 1-absorbing primary submodule of $M$.

Proof. Assume on the contrary that $N_{k}$ is a 1-absorbing primary submodule of $M$ for some $1 \leq k \leq n$. Since $N \subseteq \cup N_{i}$ is an efficient covering, $N \subseteq$ $\bigcup\left(N_{i} \cap N\right)$ is also an efficient covering. From [11, Lemma 2.1], $\left(\bigcap_{i \neq k} N_{i}\right) \cap$

$N \subseteq N_{k} \cap N$. Here, observe that $\sqrt{\left(N_{i}:_{R} M\right)}$ is a proper ideal of $R$ for all $1 \leq i \leq n$. Also, from our assumption, there is a non-unit element $a_{i} \in$ $\sqrt{\left(N_{i}:_{R} M\right)} \backslash \sqrt{\left(N_{k}:_{R} m\right)}$ for all $i \neq k$ and for all $m \in M \backslash M-\operatorname{rad}\left(N_{k}\right)$. Then there is a positive integer $n_{i}$ such that $a_{i}^{n_{i}} \in\left(N_{i}:_{R} M\right)$ for each $i \neq k$. Put 
$a=\prod_{i=1}^{k-1} a_{i}, b=\prod_{i=k+1}^{n} a_{i}$ and $n=\max \left\{n_{1}, \ldots, n_{k-1}, n_{k+1}, \ldots, n_{n}\right\}$. Now, we show that $a^{n} b^{n} m \in\left(\left(\bigcap_{i \neq k} N_{i}\right) \cap N\right) \backslash\left(N_{k} \cap N\right)$. Suppose that $a^{n} b^{n} m \in N_{k} \cap N$. Then $a^{n} b^{n} \in\left(N_{k}:_{R} m\right) \subseteq \sqrt{\left(N_{k}:_{R} m\right)}$. By Theorem $1(2), \sqrt{\left(N_{k}:_{R} m\right)}$ is a prime ideal. It implies that $a \in \sqrt{\left(N_{k}:_{R} m\right)}$ or $b \in \sqrt{\left(N_{k}:_{R} m\right)}$. Thus $a_{i} \in \sqrt{\left(N_{k}:_{R} m\right)}$ for some $i \neq k$, a contradiction. Therefore $a^{n} b^{n} m \in$ $\left(\left(\bigcap_{i \neq k} N_{i}\right) \cap N\right) \backslash\left(N_{k} \cap N\right)$ which is a contradiction. Thus $N_{k}$ is not a 1absorbing primary submodule.

Theorem 5. (1-absorbing Primary Avoidance Theorem) Let $N, N_{1}, N_{2}, \ldots, N_{n}$ $(n \geq 2)$ be submodules of $M$ such that at most two of $N_{1}, N_{2}, \ldots, N_{n}$ are not 1 absorbing primary with $N \subseteq N_{1} \cup N_{2} \cup \cdots \cup N_{n}$. If $\sqrt{\left(N_{i}:_{R} M\right)} \nsubseteq \sqrt{\left(N_{j}:_{R} m\right)}$ for all $m \in M \backslash M-\operatorname{rad}\left(N_{j}\right)$ whenever $i \neq j$, then $N \subseteq N_{k}$ for some $1 \leq k \leq n$.

Proof. Since it is clear for $n \leq 2$, suppose that $n>2$. Since any cover consisting submodules of $M$ can be reduced to an efficient one by deleting any unnecessary terms, we may assume that $N \subseteq N_{1} \cup N_{2} \cup \cdots \cup N_{n}$ is an efficient covering of submodules of $M$. From Theorem 4, it implies that no $N_{k}$ is a 1-absorbing primary submodule which contradicts with the hypothesis. Thus $N \subseteq N_{k}$ for some $1 \leq k \leq n$.

Corollary 2. Let $N$ be a proper submodule of $M$. If 1-absorbing primary avoidance theorem holds for $M$, then the 1-absorbing primary avoidance theorem holds for $M / N$.

Proof. Let $K / N, N_{1} / N, N_{2} / N, \ldots, N_{n} / N(n \geq 2)$ be submodules of $M / N$ such that at most two of $N_{1} / N, N_{2} / N, \ldots, N_{n} / N$ are not 1-absorbing primary and $K / N \subseteq N_{1} / N \cup N_{2} / N \cup \cdots \cup N_{n} / N$. Hence, $K \subseteq N_{1} \cup N_{2} \cup \cdots \cup N_{n}$ and at most two of $N_{1}, N_{2}, \ldots, N_{n}$ are not 1-absorbing primary by Corollary 1. Suppose that

$\sqrt{\left(N_{i} / N:_{R} M / N\right)} \nsubseteq \sqrt{\left(N_{j} / N:_{R} m+N\right)}$ for all $m+N \in(M / N) \backslash(M-$ $\left.\operatorname{rad}\left(N_{j} / N\right)\right)$ whenever $i \neq j$. It is easy to verify that if $\sqrt{\left(N_{i}:_{R} M\right)} \subseteq$ $\sqrt{\left(N_{j}:_{R} m\right)}$ for some $m \in M$, then $\sqrt{\left(N_{i} / N:_{R} M / N\right)} \subseteq \sqrt{\left(N_{j} / N:_{R} m+N\right)}$ for some $m+N \in M / N$. Also observe that if $m+N \in(M / N) \backslash(M / N-$ $\left.\operatorname{rad}\left(N_{j} / N\right)\right)=(M / N) \backslash\left(M-\operatorname{rad}\left(N_{j}\right) / N\right)$, then $m \in M \backslash M-\operatorname{rad}\left(N_{j}\right)$. Thus, from our assumption $\sqrt{\left(N_{i} / N:_{R} M / N\right)} \nsubseteq \sqrt{\left(N_{j} / N:_{R} m+N\right)}$ for all $m+$ $N \in(M / N) \backslash\left(M / N-\operatorname{rad}\left(N_{j} / N\right)\right)$ whenever $i \neq j$, we conclude that 
$\sqrt{\left(N_{i}:_{R} M\right)} \nsubseteq \sqrt{\left(N_{j}:_{R} m\right)}$ for all $m \in M \backslash M-\operatorname{rad}\left(N_{j}\right)$ whenever $i \neq j$. From our hypothesis and Theorem 5, we have $K \subseteq N_{k}$ for some $1 \leq k \leq n$. Consequently, $K / N \subseteq N_{k} / N$ for some $1 \leq k \leq n$; so we are done.

In view of Theorem 4 and Theorem 5, we conclude 1-absorbing primary avoidance theorem for rings.

Corollary 3. Let $I \subseteq I_{1} \cup I_{2} \cup \cdots \cup I_{n}$ be an efficient covering of ideals $I_{1}, I_{2}, \ldots, I_{n}$ of a ring $R$ where $n>2$. If $\sqrt{I_{i}} \nsubseteq \sqrt{\left(I_{j}: x\right)}$ for all $x \in R \backslash \sqrt{I_{j}}$ whenever $i \neq j$, then no $I_{i}(1 \leq i \leq n)$ is a 1-absorbing primary ideal of $R$.

Corollary 4. (1-absorbing Primary Avoidance Theorem for Rings) Let $I, I_{1}, I_{2}, \ldots, I_{n}(n \geq 2)$ be ideals of a ring $R$ such that at most two of $I_{1}, I_{2}, \ldots, I_{n}$ are not 1-absorbing primary with $I \subseteq I_{1} \cup I_{2} \cup \cdots \cup I_{n}$. If $\sqrt{I_{i}} \nsubseteq \sqrt{\left(I_{j}: x\right)}$ for all $x \in R \backslash \sqrt{I_{j}}$ whenever $i \neq j$, then $I \subseteq I_{k}$ for some $1 \leq k \leq n$.

\section{References}

[1] R. Ameri, On the prime submodules of multiplication modules. International journal of Mathematics and mathematical Sciences, 27 (2003), 1715-1724. DOI: 10.1155/S0161171203202180.

[2] M. Alkan, Y. Tiraş, On prime submodules. The Rocky Mountain Journal of Mathematics, 37(3) (2007) 709-722.

[3] S.E. Atani, F. Callıalp , U. Tekir , A Short Note on the Primary Submodules of Multiplication Modules, International Journal of Algebra, 8 (1) (2007), 381-384.

[4] A. Badawi, On 2-absorbing ideals of commutative rings, Bull. Austral. Math. Soc., 75 (2007), 417-429.

[5] A. Badawi, $n$-absorbing ideals of commutative rings and recent progress on three conjectures: a survey, Rings, Polynomials, and Modules, (2017), 33-52. DOI: 10.1007/978-3-319-65874-2_3

[6] A. Badawi, Ü. Tekir and E. Yetkin, On 2-absorbing primary ideals in commutative rings, Bull. Korean Math. Soc., 51 (4) (2014), 1163-1173.

[7] A. Badawi, E. Yetkin Celikel, On 1-absorbing primary ideals of commutative rings, Journal of Algebra and Applications, 19 (6) (2020), 2050111. DOI: $10.1142 / \mathrm{S} 021949882050111 \mathrm{X}$.

[8] A. Yousefian Darani and F. Soheilnia, On 2-absorbing and weakly 2absorbing submodules, Thai J. Math., 9 (2011), 577-584 
[9] Z. A. El-Bast and P.F. Smith, Multiplication modules. Comm. in Algebra, 16 (1988), 755-799.

[10] J. Huckaba, Rings with zero-divisors, Marcel Dekker, NewYork/ Basil,1988.

[11] C. P. Lu, Unions of prime submodules. Houston J. Math, 23 (2) (1997), 203-213

[12] C. P. Lu, M-radicals of submodules in modules, Math. Japonica, 34 (2) (1989), 211-219.

[13] R. L. McCasland and M. E. Moore, On radicals of submodules of finitely generated modules, Canad. Math. Bull., 29 (1986), 37-39.

[14] H. Mostafanasab, E. Yetkin, U. Tekir, A. Y. Darani, On 2-absorbing primary submodules of modules over comutative rings, An. S t. Univ. Ovidius Constanta, 24 (1) (2016), 335-351.

[15] S. Payrovi, B. Babaei, On 2-absorbing submodules, Algebra Colloquium, 19 (1) (2012), 913-920.

[16] P. F. Smith, Some remarks on multiplication modules, Archiv der Mathematik 50 (3) (1988), 223-235.

Ece YETKIN CELIKEL,

Department of Electrical Electronics Engineering, Faculty of Engineering,

Hasan Kalyoncu University,

Gaziantep, Turkey.

Email: ece.celikel@hku.edu.tr, yetkinece@gmail.com. 